\title{
The Design of the LED Display based on Solar Energy
}

\author{
Pei $\mathrm{LI}^{1, \mathrm{a}}$, Xuefeng BAI ${ }^{1, \mathrm{~b}}$, Hanqing $\mathrm{LI}^{1, \mathrm{~b}}$ \\ ${ }^{1}$ Beijing Information Science and Technology University, Beijing, China \\ ${ }^{a}$ Mechanical and electrical training center ${ }^{b}$ School of Automation
}

\begin{abstract}
The development of solar energy and the LED display, the superiority of the combination of them and software design of the system are discussed in this paper. Hardware design and software design are the key elements of this paper. Solar control circuit, LED display control circuit, etc are described, charge and discharge of the battery and output of the battery is controlled by STC89C52 SCM. The design of software provides driver to the related hardware circuit. The result of the experiment have achieved anticipated effects and met the design requirements.
\end{abstract}

\section{Introduction}

\subsection{The present situation and the development of solar energy}

The resources for the subsistence of the mankind is largely consuming, some of which are nearly used up. In order to protect the geo-environment and the ecology which we live on with exploiting and using the energy resource, all countries of the world have chosen the sustainable energy development strategies. Solar energy will become the new energy industry, the key development areas of development planning. Solar photovoltaic is one of the fastest growing industry of the world. In the last five years, the production of photovoltaic cells has increased steadily at an average rate of $40 \%$ per year in the world, which exceeds the development of IT industry. As a clean and renewable energy, the rapid development of the world's new energy, one of the most important direction of development. Solar energy generation industry comes into shape rapidly.(1,2 and 3)

\subsection{The meaning of solar energy application in LED display}

In recent years, more and more electronic display has been applied in many fields. It can show the changing numbers, words and graphic images. It can be used not only in the indoor environment but also in the outdoor environment, is an ideal outdoor information display media which can display text, images, two-dimensional and three-dimensional animation content has the many advantages over the projector, TV wall and LCD screen. LED panel is one kind of public information display monitor. It will be the most Microgrid display technology in the areas of display.
Solar energy can provide power for electronic displays. As a kind of the ideal public information display media, electronic display may broadcast video signal and the demonstration writing, the image such as television, video recording, VCD and so on directly. There will be a great future if we integrate solar energy utilization techniques into electronic display techniques. The combination of them produced a individual equipment that can work in many inconveniences conditions, Remote control can make screen display control more convenient and timely, changing information can move more quickly to provide convenience for the people. The solar energy power supply large and efficient use of energy saving, under cloudy conditions battery can ensure the equipment to run regularly. In places far away from civilization, such as highway, remote scenic area, The electronic display using the solar energy power supply set up bulletin boards, billboards to transfer information in time, Also low cost of investment and environmental protection give people a kind of the way that we need life--Low carbon, energy conservation, environmental protection. In this paper, After studying the principle of large number of electronic display screen, LED display based on solar energy will be designed.(4)

\section{The Working Principle and Overall Design}

As is showed in Fig1.LED display based on solar energy can be divided into three parts, the control part, battery and LED display part. Solar control principle and design system include: solar energy voltage measurement, battery charging condition and the control of charge and discharge, solar controller is key of controlling the entire system state.

General solar plate output voltage is not stable, must be converted to electrical energy stored in the storage device, cannot be applied to the load directly. solar energy 
controller plays a leading role, in the process, its performance will affect the use effect of practical application. Solar controller will control battery charge and discharge in order to prolong the service life of the battery, the controller must limit charge and discharge conditions in order to prevent the battery overcharge or deep charging.(5)

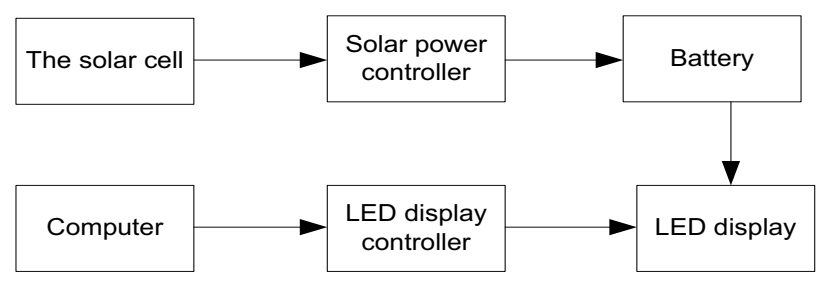

Fig.1 Solar LED display principle block diagram

\subsection{The Control of the Charge and Discharge}

In this design, the tandem type controller are selected, switch is connected between the solar cell phalanx and battery in series, Charging circuit is disconnected when the battery is full .Series switch can be used to replace the recoil diode to cut off the photovoltaic phalanx in night. Switch K1 is connected to the output of the solar cell phalanx in series. When the battery voltage is greater than the full cut off voltage, K1 will be shut down, solar panels are no longer charge to battery. Overcharge protection prevents harm to battery. As recoil diode, only when the solar cell phalanx output voltage is greater than the battery voltage, D1 will be conduction, whereas D1 ended, so as to ensure no battery will reverse discharge to solar cell phalanx back in night or rainy weather.

As the battery discharge control switch, when load current is greater than the rated load current overload or short circuit, k2 will be shut off, output overload protection and output short circuit protection. At the same time, when the battery voltage is less than the discharge voltage, K2 is also shut off to achieve the battery over discharge protection. Structure is shown in Fig. 2.

Detection control circuit will detect the battery voltage at any time, when the voltage is higher than the full cut off voltage, switch off the $\mathrm{K} 1$ to achieve over charging protection. When the voltage drops to a certain value, K1 returned charge conduction. Similarly in discharge control, when the voltage is lower than the discharge voltage, K2 will be shut off to cut off the load to achieve over discharge protection, and when the voltage is recovered to a certain value, $\mathrm{K} 2$ will be switch on to restore the discharge.(6)

PWM (pulse width modulation) charging method have been chosen in this paper, It is a very effective technology that microprocessor digital output is used to control analog circuits, widely used in measurement, communication, power control and transform in many areas. MOSFET and IGBT, the continuous improvement of controlling device, that provides PWM control technology a great foundation to promote the rapid development of the technology, have been widely applied to $\mathrm{AC} / \mathrm{DC}, \mathrm{DC} / \mathrm{DC}, \mathrm{AC} / \mathrm{AC}, \mathrm{DC} / \mathrm{AC}$ four kinds of transformation circuits.

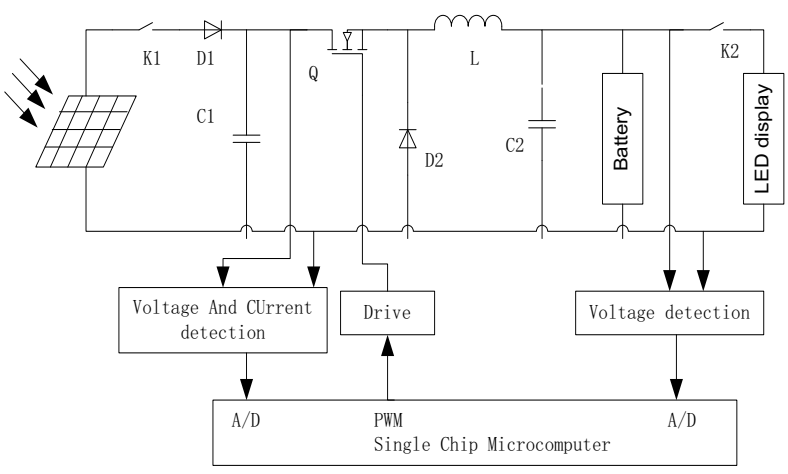

Fig.2 Solar electronic display control system structure diagram

PWM modulation way is charging battery for a period of time, and then let the battery stop charging for a period of time, such a cycle. Oxygen and hydrogen produced by the chemical reaction have time to re-organize to be absorbed, so as to reduce the battery internal pressure, make the next charging more smoothly, the battery can absorb much more power and improve the efficiency of the battery.

\subsection{Solar Control system Software Design}

The system is controlled by single-chip microcomputer. The reasonable structure of software is the key of a good performance of single chip microcomputer control system. In view of the control task to be solved, the software development procedure can be broken down into a few relatively independent modules, According to these modules connected to design the reasonable structure of the software. It will make SCM to complete the operation in an orderly way. Solar control system flow chart is shown in Fig. 3.

\subsection{LED Display Functional and Performance Requirements}

As is showed in Fig.4, LED display is consisted of control system, drive system and display device. The microprocessor control system is the core of the entire screen, which is responsible for display on electricity self-inspection and accepting information sent from PC to parses the content of agreement, according to analysis the content of the agreement to reorganize the relevant data of the agreement in order to send the right timing to drive control signal. After driver accept sequential control signal, it will drive the corresponding LED device to be the lighting or extinguishing. That will show variety of graphic left, up, down, left shutter shade, right, left or right shutters and other display effect, the PC will send correlating commands when it's time to replace them.

As a main control system, LED display control system is required to implement the following functions: 1.static and dynamic debugging. Chinese and graphic displaying functions, that can display any Chinese and a certain amount of graphic and modified by upper computer 2.The display mode control: Chinese characters or graphic can be achieved on left shift, promptly jump 
in, jump flashing, roll up into, roll down into, middle Roller shutter, left Roller shutter, right Roller shutter, both Roller shutter, left shutter, right shutter. line-by-line wipe up, line-by-line wipe down and other functions.3. Control system must have a RS-232 interface and the corresponding software, the LED display and PC are connected and formed a network through the RS-232 bus.4. Graphic and Chinese can be online updated through the application programming (TAP).5.Electricity white check function of the LED display will check whether the display work normally.

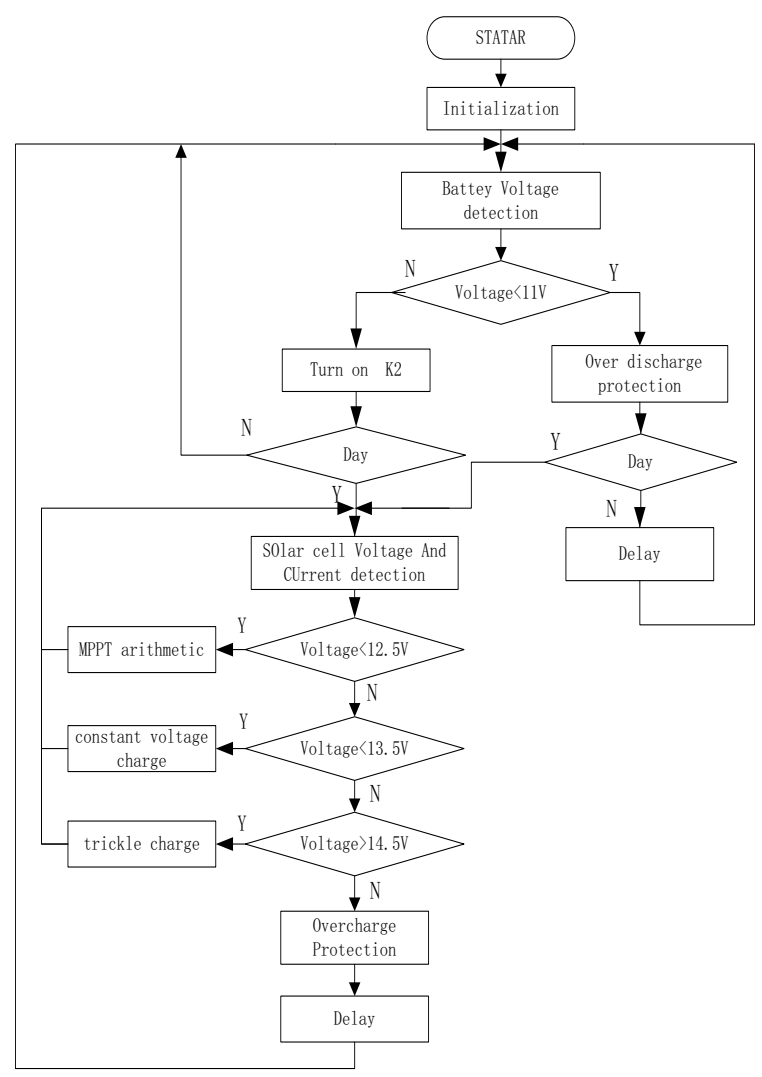

Fig.3 Solar control system flow chart

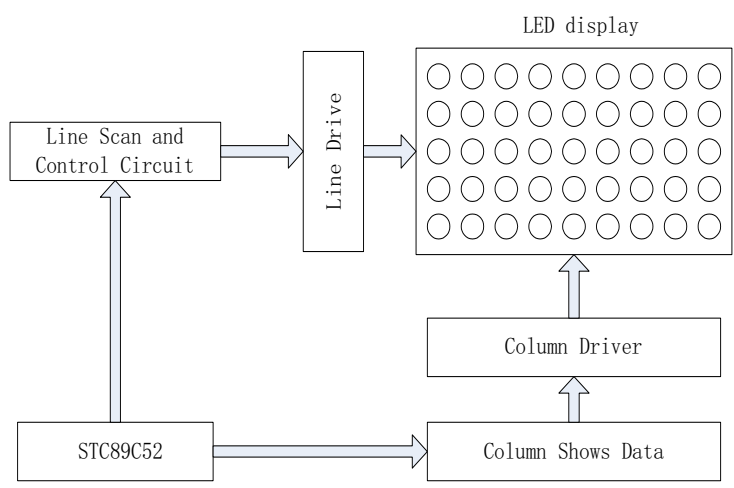

Fig. 4 LED display System structure diagram

\subsection{The control unit design}

The control unit is the core of the whole display system, the system adopts the 51 series microcontroller as the core device to process the control command and display content sent from the upper machine and PC communication. And output data to the decoding display circuit to control the LED display content and displays a status directly.(7)

The 51 series microcontroller have been selected an appropriate models as the main control chip of the control unit. On the one hand, the chip is must be convenient to programming; on the other hand, the single chip microcomputer execution speed must be quickly as far as possible. For improving the scanning speed of LED display, According to these two requirements, STC89C52RC has been chosen as control unit of the main control chip. This chip is low power consumption, high performance CMOS 8-bit MCU with $8 \mathrm{~K}$ system programmable flash memory, making it Provide high flexible and effective solution for many embedded control applications.

In addition STC89C52 can drop to $0 \mathrm{~Hz}$ static logic operation. To support two kinds of software to choose power saving mode. In the idle mode, CPU stop working and allowing the RAM, timer/counter, a serial port continue to work. In the Power-fail protection way, power lost protection mode, RAM content being saved, has been frozen, microcontroller all work stop, until the next interruption or hardware reset so far. The highest $35 \mathrm{MHZ}$ operating frequency, $6 \mathrm{t} / 12 \mathrm{t}$ is optional.

In the design of control circuit, single chip microcomputer system must work on a minimum system (refers to the MCU minimum system configuration).The minimum system includes STC89C52 external clocking circuit and reset circuit, select a certain number of 10 portals control port to control external devices and data output. According to the function to choose certain microcontroller port to add peripheral devices, the specific circuit is shown in fig5.

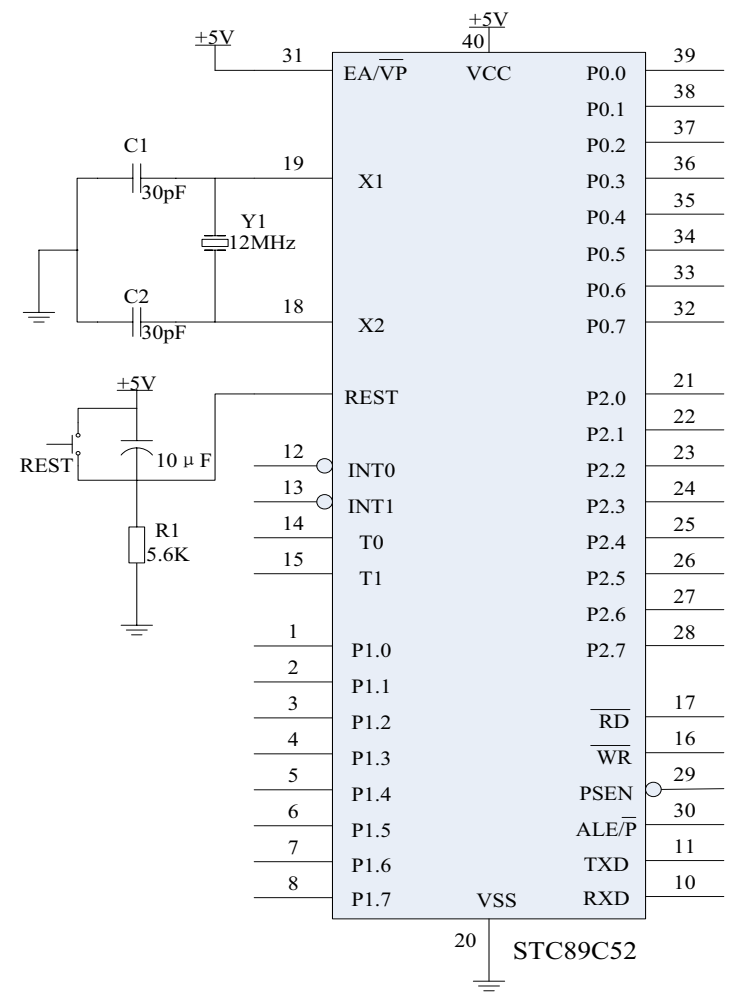

Fig.5 the controlspecific circuit 


\subsection{Communication system hardware design}

STC89C52 SCM has full duplex serial UART channels and support data serial transmission. In addition to the single chip and $\mathrm{PC}$ make communication protocol to determine the transmission speed. it will solve the problem of signal level. The RS-232C standard have ruled that the PC sending data bus TXD and receiving data bus RXD use EIA level, that is, send the number "1" on a transmission line, level is between $-3 \mathrm{~V} \sim-15 \mathrm{~V}$; send the number " 0 "on transmission, line level is between $+3 \mathrm{~V} \sim+15 \mathrm{~V}$.But the single chip microcomputer serial port is using logical TTL level, that is, the number " 1 " for $+5 \mathrm{~V}$ and " 0 " for $-5 \mathrm{~V}$, so the single chip microcomputer and computer cannot communicate directly, must transfer the TTL level with RS -232C.

In the general level conversion chip, MAX232 series chip has been used commonly in the microcontroller system with high integration, single $+5 \mathrm{~V}$ power supply work, only an external 5 small capacitance can complete conversion between RS-232C and 1vrL level now .In the display system the MAX232 is the most important hardware component of communication system .Circuit is shown in Fig. 6. $(8,9)$

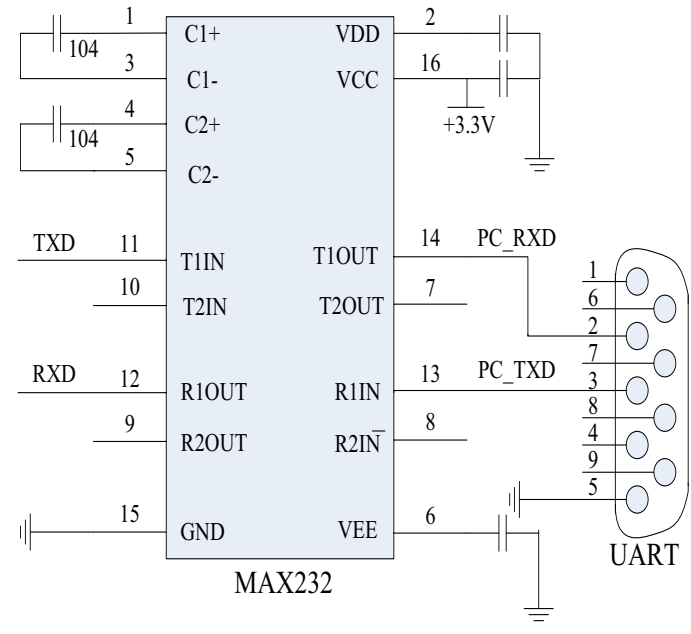

Fig.6 MAX232Circuit

\section{6 the system program design}

This program mainly includes three parts: main program, display subroutine, delay subroutine. Program flow chart is shown in figure 7.The main program mainly complete the whole hardware system initialization, mainly including the I/O port definition and initialization, each parameter used in the program setting and definition.

Call the font program and delay in turn. Display subroutine will save the bitmap data storage sequentially and line scan to remove line by line light LED. Chinese characters display on a screen, cycle show a period of time, lattice address offset 32 bytes, then show the next screen data. In the data, must think about the Chinese character modulus principle, $16 * 16$ display has row and column two kind of modulus, here is the latter. According to the modulus, first call font program, then, each hexadecimal will be send out, first call the upper part of each column.

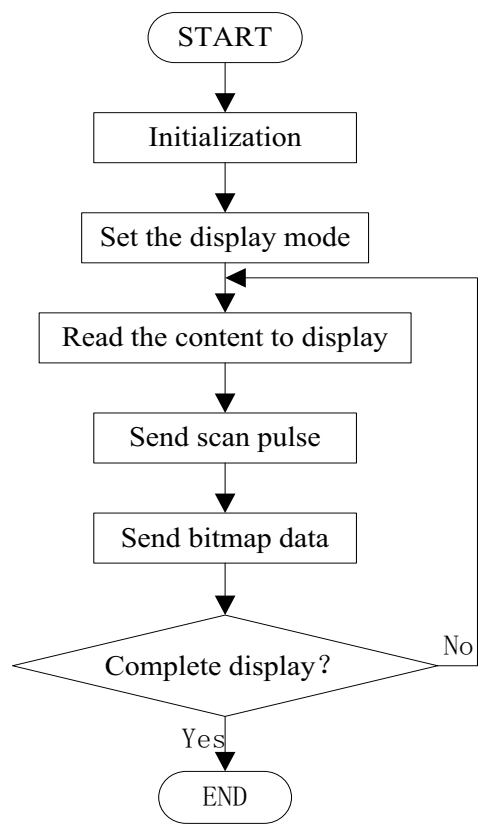

Fig. 7 program flow chart

\section{CONCLUSION}

Hardware design and software design are the key elements of this paper. Hardware includes signal acquisition circuit, solar control circuit, alarm circuit, electronic display screen circuit, etc. It controlled by STC89C52 SCM, MAX232 is used in communication system hardware design and using triode to control battery and output of the battery. The aim of the design of software is primarily to provide driver to the related hardware circuit, mainly are data acquisition program, serial communication program and the solar circuit control procedures. Programming environment uses the Keil development environment and Programming language uses the $C$ language.

The result of the experiment meets the design requirements on the whole, making the solar charging and electronic display output come true. The result shows that not only can improve flexibility of system, it also has obvious energy-saving and environmental protection effect.

In the further research, questions which needed to be studied and resolved:

1 , the remote control of the display screen.

2 , solve the problem of display the required power is too large.

\section{ACKNOWLEDGMENT}

This work was supported in part by Beijing municipal education commission science and technology project(No.KM201511232023);Beijing Higher Education Young Elite Teacher Project, 2015, (No. 71A1511136) 


\section{REFERENCES}

1. Meng-Hui Wang, Jen-Cheng yang, Xiu-Hong Li. Application of Extension Method to Selecting of Solar Power Systems [J].Department of Electrical Engineering, 2010:160-163

2. Pablo Valera, Antonio Esteban, Maria de los Reyes Carrillo. Solar Energy: Comparative analysis of solar technologies for electricity production [J]. 2003, (18): 2482-2485.

3. Menghui WANG, JenCheng YANG, XiuHong LI. Application of Extension Method to Selecting of Solar Power Systems[J].Department of Electrical Engineering, 2010:160-163.

4. Pablo Valera, Antonio Esteban, Maria de los Reyes Carrillo. solar energy: comparative analysis of solar technologies for electricity production $[\mathrm{J}] .2003$, (18):2482-2485.
5. Kitaguchi H, Miyai H, Izumi S, Kaihara A. Silicon semiconductor detectors for various nuclear radiations $[\mathrm{J}]$. Nuclear Science, IEEE Transactions on, 1996,43

6. ISLAM M R, GABBAR H A. Analysis of Microgrid Protection Strategies[C]. IEEE Smart Grid Engineering, Oshawa, ON, 2012

7. Wei QIAO, Xiao-li W ANG Intelligent lattice electronic display control system design [J]. Research and Exploration in Laboratory, 2010, 29(2):37-39.

8. Ni, Xiaohong Yan, Zhou; Dan, Liu; Zhou, Yanling. The realization of LED display system based on the embedded [J]. Telkomnika - Indonesian Journal of Electrical Engineering, 2013,11: 2626-2633

9. Hong-li CHEN. Lattice LED electronic display control system design and implementation [J]. Fujian Computer. 2010, (5):146-147. 